\title{
Association between Serum Inorganic Phosphorus Levels and Adverse Outcomes in Chronic Kidney Disease: The Fukushima CKD Cohort Study
}

\author{
Akira Oda ${ }^{1}$, Kenichi Tanaka ${ }^{1,2}$, Hirotaka Saito ${ }^{1}$, Tsuyoshi Iwasaki ${ }^{1}$, Shuhei Watanabe ${ }^{1}$, \\ Hiroshi Kimura ${ }^{1}$, Sakumi Kazama ${ }^{2}$, Michio Shimabukuro ${ }^{2,3}$, Koichi Asahi ${ }^{2,4}$, \\ Tsuyoshi Watanabe ${ }^{2}$ and Junichiro James Kazama ${ }^{1,2}$
}

\begin{abstract}
:
Objective Although an association between serum inorganic phosphorus levels and a poor prognosis has been noted in dialysis patients, these associations have been insufficiently reported in non-dialysis-dependent chronic kidney disease (NDD-CKD) patients. This study attempted to determine the association between serum inorganic phosphorus levels and adverse outcomes in Japanese NDD-CKD patients.

Methods We investigated the relationships between serum inorganic phosphorus levels and adverse outcomes, such as kidney events, cardiovascular events, and all-cause death, in Japanese NDD-CKD patients using longitudinal data from the Fukushima CKD Cohort Study with a median follow-up period of 2.8 years. The study evaluated 822 patients with NDD-CKD enrolled between June 2012 and July 2014. A kidney event was defined as a combination of doubling of the baseline serum creatinine or end-stage renal disease. Cox regression was performed to analyze the relationships of the quartile of the serum inorganic phosphorus with kidney events, cardiovascular events, and all-cause death.

Results The frequency of kidney events per 1,000 person-years exhibited a U-shaped distribution based on serum inorganic phosphorus levels, with these levels not significantly associated with an increased risk of cardiovascular events and all-cause death. A multivariable Cox regression analysis showed an increased risk of kidney events for the highest quartile of the serum inorganic phosphorus levels $(\geq 3.7 \mathrm{mg} / \mathrm{dL})$ versus the second quartile (2.9-3.2 mg/dL, hazard ratio, 3.30; 95\% confidence interval, 1.50-7.28; $p=0.003)$. There were no significant associations between the serum calcium levels and adverse outcomes.

Conclusion Serum inorganic phosphorus levels were associated with an increased risk of CKD progression in Japanese NDD-CKD patients.
\end{abstract}

Key words: Cardiovascular event, Chronic kidney disease, End-stage renal disease, Kidney event, Mortality, Phosphorus

(Intern Med Advance Publication)

(DOI: 10.2169/internalmedicine.7870-21)

\section{Introduction}

Chronic kidney disease (CKD) is associated with an increased risk of all-cause and cardiovascular death (1). Min- eral metabolism disorders are common among patients with severe CKD, including dialysis patients, and serum inorganic phosphorus and calcium abnormalities have been suggested as risk factors of these events.

Several observational studies, including studies based on

\footnotetext{
${ }^{1}$ Department of Nephrology and Hypertension, Fukushima Medical University, Japan, ${ }^{2}$ Division of Advanced Community Based Care for Lifestyle Related Diseases, Fukushima Medical University, Japan, ${ }^{3}$ Department of Diabetes, Endocrinology and Metabolism, Fukushima Medical University, Japan and ${ }^{4}$ Division of Nephrology and Hypertension, Iwate Medical University, Japan Received: May 5, 2021; Accepted: October 3, 2021; Advance Publication by J-STAGE: $\star$, $\star$ Correspondence to Dr. Kenichi Tanaka, kennichi@fmu.ac.jp
} 


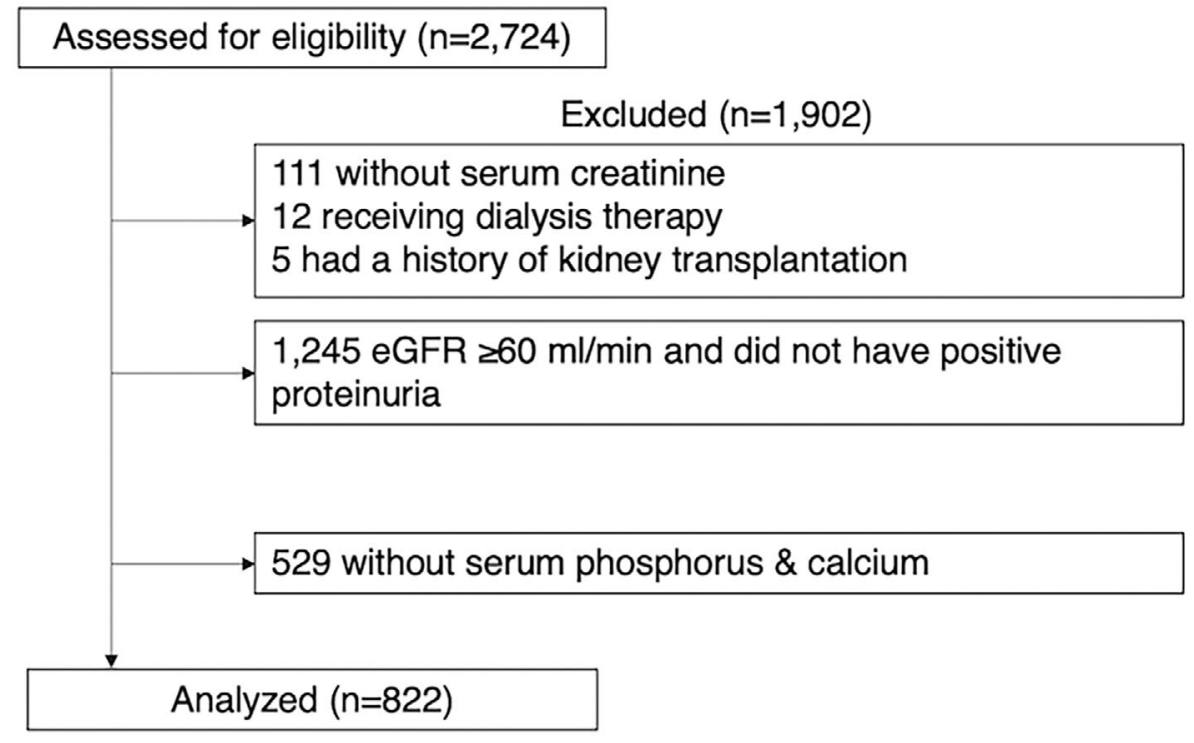

Figure 1. Participant flowchart for the present study.

data from the United States Renal Data System and the Dialysis Outcomes Practice Patterns Study, have reported associations between abnormalities in mineral metabolism, such as increased serum levels of inorganic phosphorus or calcium, and all-cause and cardiovascular mortality in dialysis patients (2-4). Recent studies have demonstrated that elevated serum levels of inorganic phosphorus were an independent risk factor for all-cause death, cardiovascular events, and CKD progression even among patients with nondialysis-dependent CKD (NDD-CKD) (5-9). Meta-analyses also demonstrated that there were independent associations between increased inorganic phosphorus levels and CKD progression and mortality in NDD-CKD patients $(10,11)$.

However, some studies have reported finding no independent associations of serum inorganic phosphorus levels with the risk for cardiovascular death or CKD progression in these patients $(12,13)$. In addition, since the serum inorganic phosphorus levels associated with the increased risk of these adverse outcomes have varied among studies, the optimal range of serum inorganic phosphorus levels in NDDCKD remains controversial.

The risk of end-stage renal disease (ESRD) is reportedly higher than that for death due to cardiovascular disease (CVD) in Japanese CKD patients, with the incidence of cardiovascular events much lower than that found in Western counterparts $(14,15)$. Since there appear to be differences in the risk of ESRD or cardiovascular events between Japanese and Western CKD patients, it is crucial to elucidate the association of serum inorganic phosphorus levels in CKD progression and other adverse events in Japanese NDD-CKD patients. However, there have been few evaluations of the relationships between serum inorganic phosphorus levels and adverse outcomes in Japanese patients.

Therefore, the present study investigated the relevance of serum inorganic phosphorus levels to CKD progression, cardiovascular events, and mortality in Japanese NDD-CKD pa- tients evaluated in the Fukushima CKD Cohort Study.

\section{Methods}

\section{Study population (Fukushima CKD Cohort)}

The Fukushima Cohort Study is a prospective survey of patient characteristics and outcomes for subjects having one or more cardiovascular risk factors, such as CKD, hypertension, diabetes, and dyslipidemia, who were followed at the Fukushima Medical University Hospital and enrolled between June 2012 and July 2014 (16). The Fukushima CKD Cohort Study is a sub-cohort of the Fukushima Cohort Study of subjects with NDD-CKD (17).

Of the originally recruited 2,724 patients in the Fukushima Cohort Study, we excluded the following subjects from this analysis: patients lacking data on serum creatinine and eGFR $\geq 60 \mathrm{~mL} / \mathrm{min} / 1.73 \mathrm{~m}^{2}$ and patients who did not have positive proteinuria at the time of registration. After the further exclusion of those without serum calcium or inorganic phosphorus data, a total of 822 participants were evaluated in the present study (Fig. 1).

This study was approved by the Ethics Committee of Fukushima Medical University (acceptance no. 1456, 2001) and carried out in accordance with the Declaration of Helsinki. The study was registered in the University Hospital Medical Information Network Clinical Trials Registry (UMIN000040848).

\section{Measurements}

Information on medication being administered at baseline as well as the history of CVD, diabetes mellitus, hypertension, and dyslipidemia were obtained from the patients' medical records or from the results of blood examinations performed at registration. Serum creatinine levels were measured by an enzyme assay method, while serum albu- 
Table 1. Patients' Characteristics by Quartiles of Serum Phosphorus Level.

\begin{tabular}{|c|c|c|c|c|c|}
\hline & \multicolumn{4}{|c|}{ Serum phosphorus (mg/dL) } & $\begin{array}{l}\mathrm{p} \text { for } \\
\text { trend }\end{array}$ \\
\hline $\mathrm{N}$ & 186 & 213 & 196 & 227 & \\
\hline Age (y) & $65(55-75)$ & $66(54-75)$ & $68(60-76)$ & $65(54-74)$ & 0.587 \\
\hline Male sex $(\%)$ & 74.7 & 65.7 & 52.6 & 37.4 & $<0.001$ \\
\hline Body mass index $\left(\mathrm{kg} / \mathrm{m}^{2}\right)$ & $24.9(22.0-26.9)$ & $23.3(20.9-25.9)$ & $23.6(21.2-27.4)$ & $23.7(20.9-26.8)$ & 0.067 \\
\hline Diabetes (\%) & 41.4 & 43.7 & 46.4 & 46.7 & 0.679 \\
\hline Dyslipidemia (\%) & 62.8 & 67.6 & 62.0 & 71.2 & 0.157 \\
\hline Cardiovascular disease $(\%)$ & 30.6 & 31.9 & 33.7 & 27.8 & 0.600 \\
\hline Systolic blood pressure (mmHg) & $127(120-139)$ & $126(118-142)$ & $136(126-149)$ & $135(122-149)$ & 0.435 \\
\hline Serum albumin $(\mathrm{g} / \mathrm{dL})$ & $4.0(3.6-4.2)$ & $4.0(3.6-4.2)$ & $3.9(3.6-4.2)$ & $3.8(3.4-4.0)$ & 0.050 \\
\hline Hemoglobin (g/dL) & $13.6(12.4-14.5)$ & $13.0(12.0-14.1)$ & $12.5(11.1-14.0)$ & $11.3(10.4-12.7)$ & $<0.001$ \\
\hline Positive proteinuria (\%) & 37.3 & 38.2 & 41.8 & 52.7 & 0.004 \\
\hline Serum phosphorus (mg/dL) & $2.6(2.4-2.8)$ & $3.1(3.0-3.2)$ & $3.4(3.3-3.5)$ & $4.0(3.8-4.4)$ & $<0.001$ \\
\hline Serum calcium (mg/dL) & $9.3(9.1-9.6)$ & $9.4(9.2-9.7)$ & $9.4(9.1-9.7)$ & $9.3(9.1-9.6)$ & 0.171 \\
\hline intact-PTH (pg/mL) & $46(33-72)$ & $43(30-69)$ & $45(32-75)$ & $62(30-159)$ & 0.104 \\
\hline
\end{tabular}

Median (25\%-75\%). eGFR: estimated glomerular filtration rate, PTH: parathyroid hormone

min, hemoglobin, inorganic phosphorus, calcium, and intactparathyroid hormone $(\mathrm{PTH})$ levels were measured according to the automated, standardized laboratory technique of the clinical laboratory of our institution. Proteinuria was detected by a urine dipstick test. Systolic blood pressure and diastolic blood pressure were measured by trained staff using a standard sphygmomanometer or an automated device with subjects in the sitting position. The body mass index was calculated as the weight $(\mathrm{kg})$ divided by the height squared (in meters, $\mathrm{m}^{2}$ ). Patients with diabetes were identified by a fasting plasma glucose concentration $\geq 126 \mathrm{mg} / \mathrm{dL}$, or a glycosylated hemoglobin (HbA1c) value (National Glycohemoglobin Standardization) $\geq 6.5 \%$, or as patients who used insulin or oral antihyperglycemic drugs. Dyslipidemia was defined as patients with either a triglyceride concentration $\geq 150 \mathrm{mg} / \mathrm{dL}$, low-density lipoprotein cholesterol concentration $\geq 140 \mathrm{mg} / \mathrm{dL}$, high-density lipoprotein cholesterol concentration $<40 \mathrm{mg} / \mathrm{dL}$, or were using antihyperlipidemic medication. CVD included myocardial infarction, angina pectoris, congestive heart failure, arrhythmias, cerebrovascular disorders, chronic arteriosclerosis obliterans, and aortic dissection.

\section{Outcomes}

Follow-up data were obtained from patients' medical records. Study endpoints were all-cause death and cardiovascular and kidney events prior to initiating the maintenance dialysis therapy. Cardiovascular events included fatal and nonfatal myocardial infarction, angina pectoris, sudden death, congestive heart failure which requires hospitalization, fatal arrhythmias, cerebrovascular disorder, chronic arteriosclerosis obliterans, and aortic dissection. Kidney events were defined as a composite of doubling of the serum creatinine or ESRD that required renal replacement therapy.

\section{Statistical analyses}

Participant characteristics were evaluated by dividing the study population into quartiles according to the serum inorganic phosphorus $(\leq 2.8,2.9-3.2,3.3-3.6, \geq 3.7 \mathrm{mg} / \mathrm{dL})$ and serum calcium ( $\leq 9.1,9.2-9.3,9.4-9.6, \geq 9.7 \mathrm{mg} / \mathrm{dL}$ ) levels. Serum calcium was corrected for a low serum albumin level (corrected calcium $=$ serum calcium $+[4$ - serum albumin $]$ ).

Data are expressed as the median and interquartile range for continuous variables and percentages for categorical data. Differences between groups were analyzed by the Kruskal-Wallis test for continuous variables and the chisquare test for categorical variables. The incidences of cardiovascular and kidney events were presented as the number of events per 1,000 person-years. Kaplan-Meier survival plots with a log-rank test and Cox proportional hazards models were used to evaluate the association of the quartile of the serum inorganic phosphorus and serum calcium levels with all-cause death and cardiovascular and kidney events. Data were analyzed using the SPSS software program, version 26 (IBM Corporation, Chicago, IL, USA).

\section{Results}

\section{Baseline characteristics}

Fig. 1 presents the patient disposition. Table 1 summarizes the baseline characteristics for the patient groups that were divided according to the quartiles of the serum inorganic phosphorus levels, while the information for the pa- 
Table 2. Patients' Characteristics by Quartiles of Serum Phosphorus Level in Men.

\begin{tabular}{|c|c|c|c|c|c|}
\hline & \multicolumn{4}{|c|}{ Serum phosphorus (mg/dL) } & \multirow{2}{*}{$\begin{array}{l}\mathrm{p} \text { for } \\
\text { trend }\end{array}$} \\
\hline & $\leq 2.8$ & $2.9-3.2$ & 3.3-3.6 & $3.7 \leq$ & \\
\hline $\mathrm{N}$ & 139 & 140 & 103 & 85 & \\
\hline Age (y) & $65(58-74)$ & $70(57-76)$ & $68(60-73)$ & $63(49-71)$ & 0.434 \\
\hline Body mass index $\left(\mathrm{kg} / \mathrm{m}^{2}\right)$ & $25.1(22.5-26.7)$ & $23.7(21.7-25.7)$ & $23.9(21.7-27.4)$ & $23.9(20.8-27.2)$ & 0.108 \\
\hline Smoking history (\%) & 71.2 & 75.7 & 68.9 & 71.8 & 0.686 \\
\hline Diabetes (\%) & 43.2 & 49.3 & 47.6 & 51.8 & 0.605 \\
\hline Dyslipidemia (\%) & 62.0 & 68.8 & 56.9 & 67.1 & 0.239 \\
\hline Cardiovascular disease (\%) & 31.7 & 39.3 & 42.7 & 31.8 & 0.219 \\
\hline Systolic blood pressure $(\mathrm{mmHg})$ & $126(120-135)$ & $130(119-143)$ & $132(118-144)$ & $132(124-145)$ & 0.584 \\
\hline Diastolic blood pressure (mmHg) & $78(70-85)$ & $79(69-81)$ & $80(73-84)$ & $81(69-87)$ & 0.315 \\
\hline Serum creatinine $(\mathrm{mg} / \mathrm{dL})$ & $1.19(0.97-1.58)$ & $1.15(1.00-1.43)$ & $1.39(1.09-2.09)$ & $2.55(1.44-3.91)$ & $<0.001$ \\
\hline $\mathrm{eGFR}\left(\mathrm{mL} / \mathrm{min} / 1.73 \mathrm{~m}^{2}\right)$ & $49.1(34.8-59.2)$ & $50.2(39.4-58.3)$ & $39.3(26.0-55.4)$ & $21.9(13.1-40.8)$ & $<0.001$ \\
\hline Serum albumin $(g / d L)$ & $4.0(3.6-4.2)$ & $4.0(3.5-4.2)$ & $3.9(3.4-4.1)$ & $3.9(3.3-4.1)$ & 0.253 \\
\hline Hemoglobin (g/dL) & $13.8(12.5-14.7)$ & $13.2(12.2-14.7)$ & $13.4(11.7-14.3)$ & $11.6(10.7-12.8)$ & $<0.001$ \\
\hline Positive proteinuria $(\%)$ & 36.9 & 40.7 & 46.1 & 63.5 & 0.002 \\
\hline Serum phosphorus (mg/dL) & $2.6(2.3-2.7)$ & $3.1(3.0-3.2)$ & $3.4(3.3-3.5)$ & $3.9(3.7-4.2)$ & $<0.001$ \\
\hline Serum calcium (mg/dL) & $9.2(9.0-9.5)$ & $9.4(9.1-9.7)$ & $9.4(9.0-9.7)$ & $9.1(8.8-9.4)$ & 0.059 \\
\hline intact-PTH (pg/mL) & $43(33-69)$ & $44(30-66)$ & $44(28-69)$ & $70(35-181)$ & 0.027 \\
\hline
\end{tabular}

Median (25\%-75\%). eGFR: estimated glomerular filtration rate, PTH: parathyroid hormone

Table 3. Patients' Characteristics by Quartiles of Serum Phosphorus Level in Women.

\begin{tabular}{|c|c|c|c|c|c|}
\hline & \multicolumn{4}{|c|}{ Serum phosphorus (mg/dL) } & \multirow{2}{*}{$\begin{array}{l}\mathrm{p} \text { for } \\
\text { trend }\end{array}$} \\
\hline & $\leq 2.8$ & $2.9-3.2$ & $3.3-3.6$ & $3.7 \leq$ & \\
\hline $\mathrm{N}$ & 47 & 73 & 93 & 142 & \\
\hline Age (y) & $64(47-77)$ & $61(45-66)$ & $72(59-78)$ & $66(57-76)$ & 0.317 \\
\hline Body mass index $\left(\mathrm{kg} / \mathrm{m}^{2}\right)$ & $22.5(19.6-27.3)$ & $21.9(20.3-27.3)$ & $22.8(20.3-27.0)$ & $23.2(20.8-27.1)$ & 0.967 \\
\hline Smoking history (\%) & 23.4 & 13.7 & 21.5 & 18.3 & 0.502 \\
\hline Diabetes $(\%)$ & 36.2 & 32.9 & 45.2 & 43.7 & 0.317 \\
\hline Dyslipidemia (\%) & 65.2 & 65.3 & 67.8 & 73.8 & 0.508 \\
\hline Cardiovascular disease (\%) & 27.7 & 17.8 & 23.7 & 25.4 & 0.567 \\
\hline Systolic blood pressure (mmHg) & $137(123-151)$ & $126(108-143)$ & $141(131-158)$ & $137(122-154)$ & 0.654 \\
\hline Diastolic blood pressure (mmHg) & $79(73-94)$ & $72(66-84)$ & $79(67-86)$ & $76(66-85)$ & 0.343 \\
\hline Serum creatinine $(\mathrm{mg} / \mathrm{dL})$ & $0.92(0.78-1.07)$ & $0.88(0.70-1.12)$ & $0.83(0.70-1.21)$ & $1.26(0.80-2.94)$ & 0.073 \\
\hline $\mathrm{eGFR}\left(\mathrm{mL} / \mathrm{min} / 1.73 \mathrm{~m}^{2}\right)$ & $49.7(41.2-55.9)$ & $49.1(40.7-69.0)$ & $52.1(34.5-62.3)$ & $35.1(12.8-53.4)$ & 0.045 \\
\hline Serum albumin $(g / d L)$ & $3.9(3.5-4.3)$ & $3.8(3.6-4.2)$ & $3.9(3.6-4.2)$ & $3.8(3.4-3.9)$ & 0.369 \\
\hline Hemoglobin (g/dL) & $13.2(11.4-13.5)$ & $12.7(10.8-13.8)$ & $11.6(10.8-13.8)$ & $11.2(10.0-12.3)$ & 0.003 \\
\hline Positive proteinuria (\%) & 30.4 & 33.3 & 36.8 & 46.0 & 0.140 \\
\hline Serum phosphorus (mg/dL) & $2.7(2.5-2.8)$ & $3.1(3.0-3.2)$ & $3.4(3.3-3.5)$ & $4.1(3.8-4.4)$ & $<0.001$ \\
\hline Serum calcium $(\mathrm{mg} / \mathrm{dL})$ & $9.5(9.3-9.7)$ & $9.4(9.3-9.6)$ & $9.4(9.2-9.6)$ & $9.4(9.2-9.7)$ & 0.457 \\
\hline intact-PTH (pg/mL) & $50(40-85)$ & $44(31-78)$ & $51(37-82)$ & $59(28-126)$ & 0.880 \\
\hline
\end{tabular}

Median (25\%-75\%). eGFR: estimated glomerular filtration rate, PTH: parathyroid hormone

tient groups divided according to the quartiles of the serum calcium levels are shown in supplemental Table S1. There were no significant differences in the age, diabetes, dyslipidemia, or history of CVD across quartiles of serum inorganic phosphorus. Subjects with higher serum inorganic phosphorus levels had a significantly lower eGFR and lower hemoglobin than those with lower serum inorganic phosphorus levels. In contrast, subjects with higher serum calcium levels had a higher eGFR, higher hemoglobin, and lower PTH value than those with lower serum calcium levels.
There were 37 hyperphosphatemia patients $(4.5 \%$ of the total) with a serum inorganic phosphorus level $\geq 4.5 \mathrm{mg} / \mathrm{dL}$ and 25 hypercalcemia patients (3\% of the total) with a serum calcium level $\geq 10 \mathrm{mg} / \mathrm{dL}$. Baseline patients' characteristics by quartiles of serum inorganic phosphorus level are shown in men and women, separately (Table 2, 3).

\section{Serum inorganic phosphorus levels and adverse outcomes}

Over a median follow-up period of 2.8 years, 46 patients 
Table 4. Associations of Serum Phosphorus Levels with All-cause Death, Cardiovascular Event, and Kidney Event in Non-dialysis Dependent CKD Patients.

\begin{tabular}{|c|c|c|c|c|c|c|c|c|c|}
\hline & \multirow{2}{*}{$\begin{array}{c}\text { Incident rate } \\
(/ 1,000 \\
\text { person-years })\end{array}$} & \multicolumn{2}{|c|}{ Univariate } & \multicolumn{2}{|c|}{ Model 1} & \multicolumn{2}{|c|}{ Model 2} & \multicolumn{2}{|c|}{ Model 3} \\
\hline & & $\begin{array}{c}\mathrm{HR} \\
(95 \% \mathrm{CI})\end{array}$ & $\mathrm{p}$ & $\begin{array}{c}\mathrm{HR} \\
(95 \% \mathrm{CI})\end{array}$ & $\mathrm{p}$ & $\begin{array}{c}\mathrm{HR} \\
(95 \% \mathrm{CI})\end{array}$ & $\mathrm{p}$ & $\begin{array}{c}\mathrm{HR} \\
(95 \% \mathrm{CI})\end{array}$ & $\mathrm{p}$ \\
\hline \multicolumn{10}{|l|}{ All-cause death } \\
\hline \multicolumn{10}{|l|}{ Serum phosphorus } \\
\hline$<2.8 \mathrm{mg} / \mathrm{dL}$ & 20.4 & $\begin{array}{c}0.97 \\
(0.44-2.18)\end{array}$ & 0.949 & $\begin{array}{c}1.02 \\
(0.46-2.28)\end{array}$ & 0.964 & $\begin{array}{c}1.07 \\
(0.48-2.39)\end{array}$ & 0.875 & $\begin{array}{c}1.20 \\
(0.52-2.77)\end{array}$ & 0.672 \\
\hline $2.9-3.2 \mathrm{mg} / \mathrm{dL}$ & 20.8 & $\begin{array}{c}1.00 \\
\text { [reference] }\end{array}$ & & $\begin{array}{c}1.00 \\
\text { [reference] }\end{array}$ & & $\begin{array}{c}1.00 \\
\text { [reference] }\end{array}$ & & $\begin{array}{c}1.00 \\
\text { [reference] }\end{array}$ & \\
\hline $3.3-3.6 \mathrm{mg} / \mathrm{dL}$ & 12.7 & $\begin{array}{c}0.60 \\
(0.24-1.51)\end{array}$ & 0.279 & $\begin{array}{c}0.66 \\
(0.26-1.66)\end{array}$ & 0.374 & $\begin{array}{c}0.64 \\
(0.25-1.61)\end{array}$ & 0.337 & $\begin{array}{c}0.58 \\
(0.22-1.52)\end{array}$ & 0.266 \\
\hline$\geq 3.7 \mathrm{mg} / \mathrm{dL}$ & 24.7 & $\begin{array}{c}1.17 \\
(0.56-2.47)\end{array}$ & 0.674 & $\begin{array}{c}1.49 \\
(0.68-3.24)\end{array}$ & 0.321 & $\begin{array}{c}1.53 \\
(0.68-3.43)\end{array}$ & 0.302 & $\begin{array}{c}1.26 \\
(0.55-2.91)\end{array}$ & 0.587 \\
\hline per $1-\mathrm{mg} / \mathrm{dL}$ increase & & $\begin{array}{c}1.08 \\
(0.68-1.69)\end{array}$ & 0.754 & $\begin{array}{c}1.23 \\
(0.75-2.01)\end{array}$ & 0.423 & $\begin{array}{c}1.20 \\
(0.72-2.00)\end{array}$ & 0.489 & $\begin{array}{c}1.00 \\
(0.59-1.70)\end{array}$ & 0.988 \\
\hline \multicolumn{10}{|l|}{ Cardiovascular event } \\
\hline \multicolumn{10}{|l|}{ Serum phosphorus } \\
\hline$<2.8 \mathrm{mg} / \mathrm{dL}$ & 20.9 & $\begin{array}{c}1.44 \\
(0.60-3.48)\end{array}$ & 0.416 & $\begin{array}{c}1.45 \\
(0.60-3.48)\end{array}$ & 0.416 & $\begin{array}{c}0.32 \\
(0.54-3.21)\end{array}$ & 0.543 & $\begin{array}{c}1.36 \\
(0.55-3.39)\end{array}$ & 0.507 \\
\hline $2.9-3.2 \mathrm{mg} / \mathrm{dL}$ & 14.6 & $\begin{array}{c}1.00 \\
\text { [reference] }\end{array}$ & & $\begin{array}{c}1.00 \\
\text { [reference] }\end{array}$ & & $\begin{array}{c}1.00 \\
\text { [reference] }\end{array}$ & & $\begin{array}{c}1.00 \\
\text { [reference] }\end{array}$ & \\
\hline $3.3-3.6 \mathrm{mg} / \mathrm{dL}$ & 24.3 & $\begin{array}{c}1.67 \\
(0.72-3.91)\end{array}$ & 0.235 & $\begin{array}{c}2.02 \\
(0.86-4.74)\end{array}$ & 0.107 & $\begin{array}{c}1.63 \\
(0.69-3.83)\end{array}$ & 0.267 & $\begin{array}{c}1.70 \\
(0.72-4.04)\end{array}$ & 0.229 \\
\hline$\geq 3.7 \mathrm{mg} / \mathrm{dL}$ & 28.8 & $\begin{array}{c}1.97 \\
(0.88-4.43)\end{array}$ & 0.099 & $\begin{array}{c}3.08 \\
(1.34-7.09)\end{array}$ & 0.008 & $\begin{array}{c}1.72 \\
(0.71-4.18)\end{array}$ & 0.229 & $\begin{array}{c}1.87 \\
(0.73-4.77)\end{array}$ & 0.191 \\
\hline per $1-\mathrm{mg} / \mathrm{dL}$ increase & & $\begin{array}{c}1.42 \\
(0.95-2.12)\end{array}$ & 0.092 & $\begin{array}{c}1.90 \\
(1.25-2.90)\end{array}$ & 0.003 & $\begin{array}{c}1.20 \\
(0.78-1.85)\end{array}$ & 0.411 & $\begin{array}{c}1.17 \\
(0.75-1.85)\end{array}$ & 0.488 \\
\hline \multicolumn{10}{|l|}{ Kidney event } \\
\hline \multicolumn{10}{|l|}{ Serum phosphorus } \\
\hline$<2.8 \mathrm{mg} / \mathrm{dL}$ & 28.6 & $\begin{array}{c}2.20 \\
(0.93-5.19)\end{array}$ & 0.072 & $\begin{array}{c}2.12 \\
(0.90-5.00)\end{array}$ & 0.086 & $\begin{array}{c}2.19 \\
(0.92-5.20)\end{array}$ & 0.077 & $\begin{array}{c}1.73 \\
(0.72-4.12)\end{array}$ & 0.218 \\
\hline $2.9-3.2 \mathrm{mg} / \mathrm{dL}$ & 12.9 & $\begin{array}{c}1.00 \\
\text { [reference] }\end{array}$ & & $\begin{array}{c}1.00 \\
\text { [reference] }\end{array}$ & & $\begin{array}{c}1.00 \\
\text { [reference] }\end{array}$ & & $\begin{array}{c}1.00 \\
\text { [reference] }\end{array}$ & \\
\hline $3.3-3.6 \mathrm{mg} / \mathrm{dL}$ & 43.1 & $\begin{array}{c}3.34 \\
(1.49-7.47)\end{array}$ & 0.003 & $\begin{array}{c}3.69 \\
(1.65-8.26)\end{array}$ & 0.002 & $\begin{array}{c}2.66 \\
(1.19-5.97)\end{array}$ & 0.017 & $\begin{array}{c}1.98 \\
(0.87-4.52)\end{array}$ & 0.104 \\
\hline$\geq 3.7 \mathrm{mg} / \mathrm{dL}$ & 103.5 & $\begin{array}{c}8.09 \\
(3.86-16.98)\end{array}$ & $<0.001$ & $\begin{array}{c}10.70 \\
(5.03-22.75)\end{array}$ & $<0.001$ & $\begin{array}{c}4.03 \\
(1.87-8.66)\end{array}$ & $<0.001$ & $\begin{array}{c}3.30 \\
(1.50-7.28)\end{array}$ & 0.003 \\
\hline per $1-\mathrm{mg} / \mathrm{dL}$ increase & & $\begin{array}{c}2.80 \\
(2.21-3.55)\end{array}$ & $<0.001$ & $\begin{array}{c}3.42 \\
(2.67-4.39)\end{array}$ & $<0.001$ & $\begin{array}{c}1.61 \\
(1.22-2.13)\end{array}$ & 0.001 & $\begin{array}{c}1.55 \\
(1.15-2.09)\end{array}$ & 0.004 \\
\hline
\end{tabular}

HR: hazard ratio, CI: confidence interval. Model 1, adjusted for age and sex. Model 2, adjusted for Model 1 covariates plus smoking history, diabetes, history of cardiovascular disease, and eGFR. Model 3, adjusted for Model 2 covariates plus body mass index, systolic blood pressure, proteinuria positive, serum albumin, hemoglobin, and serum calcium.

died, and there were 50 cardiovascular events and 102 kidney events. Of these patients, 35 exhibited doubling of the serum creatinine level, while 67 progressed to ESRD requiring dialysis. When the incidence rates were stratified according to the serum inorganic phosphorus categories at baseline, U-shaped relationships were observed for the kidney events (Table 4). A significant difference $(p<0.001)$ was found in the incidence of kidney events among the NDDCKD patients with different serum inorganic phosphorus levels at baseline but not for the incidence of cardiovascular events and all-cause death (Fig. 2A, B and C). The multivariate Cox regression analysis results showed that an elevated serum inorganic phosphorus level was significantly as- sociated with an increased risk of kidney events, with the lowest risk shown for a serum inorganic phosphorus level of 2.9-3.2 $\mathrm{mg} / \mathrm{dL}$. When compared to the reference level of 2.9-3.2 $\mathrm{mg} / \mathrm{dL}$, the adjusted hazard ratio for the kidney events was 1.73 (95\% confidence interval [CI]; 0.72-4.12) for serum inorganic phosphorus $\leq 2.8 \mathrm{mg} / \mathrm{dL}, 1.98$ (95\% CI; $0.87-4.52)$ for $3.3-3.6 \mathrm{mg} / \mathrm{dL}$ and 3.30 (95\% CI; $1.50-7.28)$ for $\geq 3.7 \mathrm{mg} / \mathrm{dL}$ (Model 3 in Table 4). A 1-mg/dL increase in the serum inorganic phosphorus was associated with an adjusted hazard ratio of 1.55 (95\% CI; 1.15-2.09) for kidney events. There were no significant associations between the serum inorganic phosphorus levels at baseline and the risk of cardiovascular events or all-cause death. 


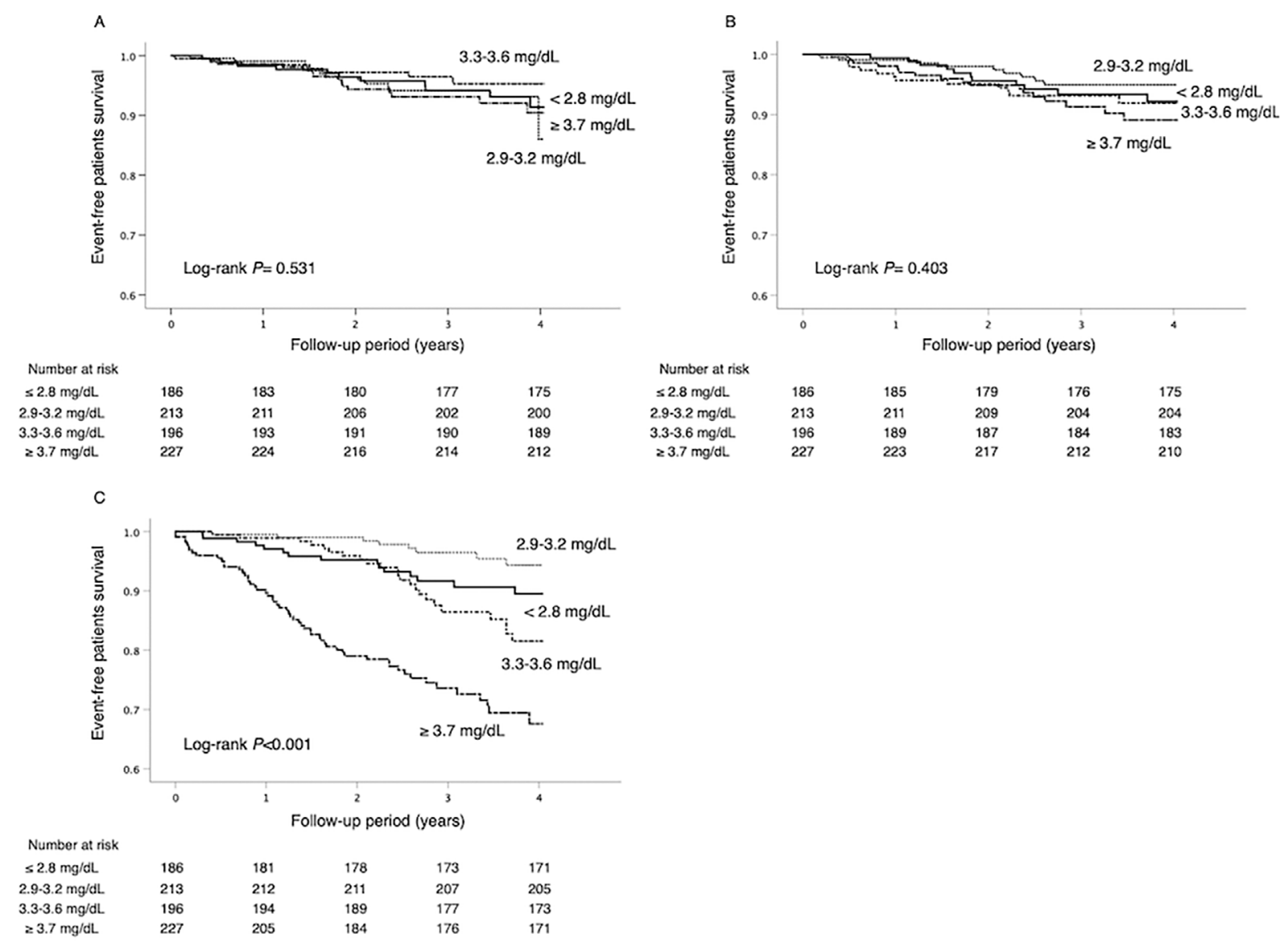

Figure 2. Kaplan-Meier curves for the incidence of all-cause death (A), cardiovascular events (B), and kidney events $(C)$, in accordance with the serum inorganic phosphorus levels at baseline in nondialysis-dependent CKD.

There were significant differences in sex distributions across quartiles of serum inorganic phosphorus levels (Table 1), so associations of serum inorganic phosphorus levels with adverse outcomes were evaluated in men and women, separately (Table 5,6). Significant association was observed between kidney events and elevated serum inorganic phosphorus levels in men. U-shaped relationships were observed in the incidences of kidney events, but due to limited number of events (no kidney events were observed in subjects with a serum inorganic phosphorus level of $2.9-3.2 \mathrm{mg} / \mathrm{dL}$ in women), Cox regression could not be performed for kidney events in women. There were no significant associations between the serum inorganic phosphorus levels and the risk of cardiovascular events and all-cause death in women, but there was a marginal significant difference between the serum inorganic phosphorus levels and the risk of cardiovascular events in men (hazard ratio 3.22, 95\% CI; 1.17-885, p= 0.023 , for $\geq 3.7 \mathrm{mg} / \mathrm{dL}$, compared to the reference level of $2.9-3.2 \mathrm{mg} / \mathrm{dL}$ ).

Kidney events were evaluated in cases with doubling of the serum creatinine and ESRD, separately (supplemental Table S2). Due to the limited number of events, multivariate adjustment was not performed for doubling of the serum creatinine level, but the serum inorganic phosphorus levels had a significant association with ESRD.

\section{Serum calcium levels and adverse outcomes}

Significant differences were found in the incidence of cardiovascular $(p=0.008)$ and kidney events $(p=0.004)$ among patients with different serum calcium levels but not in the incidence of all-cause death (Supplemental Fig. S1). Although a univariate analysis showed that the risks of cardiovascular and kidney events were significantly higher with a serum calcium level of $\leq 9.1 \mathrm{mg} / \mathrm{dL}$ than with a serum calcium level of 9.4-9.6 mg/dL, the significant associations between the serum calcium levels and the risk of cardiovascular and kidney events disappeared after multivariate adjustment for confounding factors (supplemental Table S3).

\section{Discussion}

The present study investigated serum inorganic phosphorus and calcium associations with mortality and cardiovascular and kidney events in Japanese NDD-CKD patients and demonstrated that serum inorganic phosphorus levels were independently associated with kidney events but not with mortality or cardiovascular events. U-shaped relationships were observed between the serum inorganic phosphorus levels and the incidence of kidney events, with the best outcomes seen with serum inorganic phosphorus levels of 2.9- 
Table 5. Associations of Serum Phosphorus Levels with All-cause Death, Cardiovascular Event, and Kidney Event in Nondialysis Dependent CKD Patients in Men.

\begin{tabular}{|c|c|c|c|c|c|c|c|}
\hline & \multirow{2}{*}{$\begin{array}{l}\text { Incident rate } \\
(/ 1,000 \\
\text { person-years })\end{array}$} & \multicolumn{2}{|l|}{ Univariate } & \multicolumn{2}{|l|}{ Model 1} & \multicolumn{2}{|l|}{ Model 2} \\
\hline & & HR $(95 \% \mathrm{CI})$ & $\mathrm{p}$ & HR $(95 \% \mathrm{CI})$ & $\mathrm{p}$ & HR $(95 \% \mathrm{CI})$ & $\mathrm{p}$ \\
\hline \multicolumn{8}{|l|}{ All-cause death } \\
\hline \multicolumn{8}{|l|}{ Serum phosphorus } \\
\hline$<2.8 \mathrm{mg} / \mathrm{dL}$ & 17.2 & $0.78(0.29-2.10)$ & 0.626 & $0.90(0.34-2.43)$ & 0.840 & $0.97(0.36-2.64)$ & 0.958 \\
\hline $2.9-3.2 \mathrm{mg} / \mathrm{dL}$ & 21.6 & 1.00 [reference] & & 1.00 [reference] & & 1.00 [reference] & \\
\hline $3.3-3.6 \mathrm{mg} / \mathrm{dL}$ & 17.6 & $0.78(0.26-2.34)$ & 0.662 & $0.86(0.29-2.57)$ & 0.786 & $0.80(0.27-2.40)$ & 0.688 \\
\hline$\geq 3.7 \mathrm{mg} / \mathrm{dL}$ & 48.0 & $2.15(0.87-5.31)$ & 0.096 & $2.57(1.04-6.36)$ & 0.041 & $2.53(0.96-6.68)$ & 0.060 \\
\hline per $1-\mathrm{mg} / \mathrm{dL}$ increase & & $2.03(1.15-3.56)$ & 0.014 & $2.03(1.16-3.55)$ & 0.013 & $1.82(1.01-3.29)$ & 0.048 \\
\hline \multicolumn{8}{|l|}{ Cardiovascular event } \\
\hline \multicolumn{8}{|l|}{ Serum phosphorus } \\
\hline$<2.8 \mathrm{mg} / \mathrm{dL}$ & 25.3 & $1.49(0.57-3.92)$ & 0.417 & $1.60(0.61-4.21)$ & 0.341 & $1.54(0.96-1.02)$ & 0.390 \\
\hline $2.9-3.2 \mathrm{mg} / \mathrm{dL}$ & 17.1 & 1.00 [reference] & & 1.00 [reference] & & 1.00 [reference] & \\
\hline $3.3-3.6 \mathrm{mg} / \mathrm{dL}$ & 29.3 & $1.73(0.63-4.76)$ & 0.291 & $1.82(0.66-5.02)$ & 0.248 & $1.58(0.57-4.37)$ & 0.378 \\
\hline$\geq 3.7 \mathrm{mg} / \mathrm{dL}$ & 67.0 & $3.97(1.58-9.95)$ & 0.03 & $4.41(1.76-11.1)$ & 0.002 & $3.22(1.17-8.85)$ & 0.023 \\
\hline per $1-\mathrm{mg} / \mathrm{dL}$ increase & & $2.37(1.46-3.85)$ & $<0.001$ & $2.35(1.47-3.78)$ & $<0.001$ & $1.64(1.02-2.64)$ & 0.043 \\
\hline \multicolumn{8}{|l|}{ Kidney event } \\
\hline \multicolumn{8}{|l|}{ Serum phosphorus } \\
\hline$<2.8 \mathrm{mg} / \mathrm{dL}$ & 33.1 & $1.69(0.700-4.08)$ & 0.243 & $1.71(0.71-4.12)$ & 0.235 & $1.44(0.59-3.51)$ & 0.418 \\
\hline $2.9-3.2 \mathrm{mg} / \mathrm{dL}$ & 19.5 & 1.00 [reference] & & 1.00 [reference] & & 1.00 [reference] & \\
\hline $3.3-3.6 \mathrm{mg} / \mathrm{dL}$ & 32.1 & $1.64(0.63-4.26)$ & 0.308 & $1.66(0.638-4.293)$ & 0.956 & $1.03(0.39-2.69)$ & 0.017 \\
\hline$\geq 3.7 \mathrm{mg} / \mathrm{dL}$ & 193.9 & $10.19(4.67-22.09)$ & $<0.001$ & $10.37(4.77-22.54)$ & $<0.001$ & $3.33(1.46-7.62)$ & 0.004 \\
\hline per $1-\mathrm{mg} / \mathrm{dL}$ increase & & $4.31(3.13-5.94)$ & $<0.001$ & $4.32(3.14-5.95)$ & $<0.001$ & $2.20(1.49-3.25)$ & $<0.001$ \\
\hline
\end{tabular}

HR: hazard ratio, CI: confidence interval. Model 1, adjusted for age. Model 2, adjusted for Model 1 covariates plus smoking history, diabetes, history of cardiovascular disease, and eGFR.

$3.2 \mathrm{mg} / \mathrm{dL}$. A serum inorganic phosphorus level of $\geq 3.7 \mathrm{mg} /$ dL was significantly associated with an increased risk of kidney events compared to the reference level of 2.9-3.2 mg/ dL.

There were no independent associations between the serum calcium levels and adverse outcomes in the present study. Higher serum calcium levels would be expected to contribute to adverse outcomes, due to precipitation of calcium-phosphorus product in vessels, causing vascular calcification. Indeed, an elevated serum calcium level was reportedly associated with mortality in dialysis patients (2-4). However, several recent studies have reported the association between low serum calcium levels and CKD progression in patients with NDD-CKD $(18,19)$. Given that additional residual confounding factors, such as vitamin D deficiency, may influence these results in addition to sample size or observation period, further research is still needed to clarify the effects of serum calcium on CKD progression and mortality in NDD-CKD.

Previous studies have identified a relationship between serum inorganic phosphorus levels and cardiovascular events, kidney events, and all-cause mortality in NDD-CKD patients $(6,7,11,20,21)$. These studies reported that serum inorganic phosphorus levels of $\geq 3.5 \mathrm{mg} / \mathrm{dL}$ were associated with these adverse outcomes. Although the serum inorganic phosphorus levels that contributed to this increased risk for these adverse outcomes have varied among studies, the cut-off values of serum inorganic phosphorus reported in the studies were similar to that found in the present study. In addition, the results of the present study were similar to those reported in prior studies.

It should be noted, however, that even when serum inorganic phosphorus levels are within the reference range, they can potentially cause adverse kidney outcomes. Since our present study found that only $4.5 \%$ of the population had hyperphosphatemia $(\geq 4.5 \mathrm{mg} / \mathrm{dL})$, this indicates that the majority of the patients in the highest quartile of the serum inorganic phosphorus levels had serum inorganic phosphorus levels that were within the reference range. In addition, an association between the inorganic phosphorus levels and kidney events has also been reported in Japanese NDD-CKD patients (22), with an inorganic phosphorus level of $\geq 3.4$ $\mathrm{mg} / \mathrm{dL}$ shown to be a risk factor for CKD progression to ESRD. Therefore, elevated serum inorganic phosphorus levels that are still within the reference range may carry a risk of adverse kidney outcomes in NDD-CKD patients. The present results suggest the importance of serum inorganic phosphorus management in CKD patients with regard to kidney outcomes. However, a more detailed evaluation is still required to verify the optimal range of serum inorganic phosphorus levels.

The present study found that there were marginal signifi- 
Table 6. Associations of Serum Phosphorus Levels with All-cause Death, Cardiovascular Event, and Kidney Event in Non-dialysis Dependent CKD Patients in Women.

\begin{tabular}{|c|c|c|c|c|c|c|c|}
\hline & \multirow{2}{*}{$\begin{array}{c}\text { Incident rate } \\
\quad(/ 1,000 \\
\text { person-years })\end{array}$} & \multicolumn{2}{|c|}{ Univariate } & \multicolumn{2}{|l|}{ Model 1} & \multicolumn{2}{|l|}{ Model 2} \\
\hline & & $\operatorname{HR}(95 \% \mathrm{CI})$ & $\mathrm{p}$ & $\mathrm{HR}(95 \% \mathrm{CI})$ & $\mathrm{p}$ & HR $(95 \%$ CI $)$ & $\mathrm{p}$ \\
\hline \multicolumn{8}{|l|}{ All-cause death } \\
\hline \multicolumn{8}{|l|}{ Serum phosphorus } \\
\hline$<2.8 \mathrm{mg} / \mathrm{dL}$ & 30.1 & $1.58(0.39-6.31)$ & 0.520 & $1.59(0.40-6.37)$ & 0.511 & $1.39(0.34-5.78)$ & 0.648 \\
\hline $2.9-3.2 \mathrm{mg} / \mathrm{dL}$ & 19.2 & 1.00 [reference] & & 1.00 [reference] & & 1.00 [reference] & \\
\hline $3.3-3.6 \mathrm{mg} / \mathrm{dL}$ & 7.5 & $0.39(0.07-2.13)$ & 0.277 & $0.34(0.06-1.86)$ & 0.213 & $0.34(0.06-1.88)$ & 0.218 \\
\hline$\geq 3.7 \mathrm{mg} / \mathrm{dL}$ & 12.5 & $0.65(0.18-2.43)$ & 0.524 & $0.59(0.16-2.21)$ & 0.434 & $0.61(0.16-2.29)$ & 0.463 \\
\hline per $1-\mathrm{mg} / \mathrm{dL}$ increase & & $0.48(0.19-1.20)$ & 0.117 & $0.44(0.17-1.14)$ & 0.090 & $0.49(0.19-1.25)$ & 0.136 \\
\hline \multicolumn{8}{|l|}{ Cardiovascular event } \\
\hline \multicolumn{8}{|l|}{ Serum phosphorus } \\
\hline$<2.8 \mathrm{mg} / \mathrm{dL}$ & 7.6 & $0.80(0.07-8.77)$ & 0.852 & $0.78(0.07-8.57)$ & 0.835 & $3.10(0.23-40.94)$ & 0.391 \\
\hline $2.9-3.2 \mathrm{mg} / \mathrm{dL}$ & 9.7 & 1.00 [reference] & & 1.00 [reference] & & 1.00 [reference] & \\
\hline $3.3-3.6 \mathrm{mg} / \mathrm{dL}$ & 19.1 & $1.99(0.39-10.28)$ & 0.409 & $1.57(0.30-8.12)$ & 0.591 & $2.49(0.41-15.02)$ & 0.318 \\
\hline$\geq 3.7 \mathrm{mg} / \mathrm{dL}$ & 10.1 & $1.03(0.19-5.61)$ & 0.975 & $0.87(0.16-4.78)$ & 0.876 & $0.56(0.08-3.84)$ & 0.551 \\
\hline per $1-\mathrm{mg} / \mathrm{dL}$ increase & & $1.10(0.47-2.58)$ & 0.828 & $1.09(0.44-2.72)$ & 0.859 & $0.37(0.12-1.10)$ & 0.072 \\
\hline
\end{tabular}

\begin{tabular}{|c|c|}
\hline Kidney event & \\
\hline Serum phosphoru & \\
\hline$<2.8 \mathrm{mg} / \mathrm{dL}$ & 15.2 \\
\hline $2.9-3.2 \mathrm{mg} / \mathrm{dL}$ & 0 \\
\hline $3.3-3.6 \mathrm{mg} / \mathrm{dL}$ & 55.2 \\
\hline$\geq 3.7 \mathrm{mg} / \mathrm{dL}$ & 62.0 \\
\hline
\end{tabular}

N/A
1.00 [reference]
N/A
N/A
$2.56(1.74-3.78)$
N/A
1.00 [reference]
N/A
N/A

$2.56(1.74-3.78) \quad<0.001 \quad 2.50(1.68-3.70)$
N/A
1.00 [reference]
N/A
N/A

$<0.001 \quad 1.23(0.80-1.88)$

0.349

HR: hazard ratio, CI: confidence interval, N/A: not applicable. Model 1, adjusted for age. Model 2, adjusted for Model 1 covariates plus smoking history, diabetes, history of cardiovascular disease, and eGFR.

cant associations observed between serum inorganic phosphorus levels at baseline and the risk of cardiovascular events only in men, with no significant associations with the risk of cardiovascular events or all-cause mortality noted in the whole cohort. Other studies have reported detecting associations between higher serum inorganic phosphorus levels and the risk of CVD events and all-cause death in NDDCKD patients $(5,7,11,13,20,21,23)$. However, since the incidence of cardiovascular events or all-cause death is reportedly lower in Japanese NDD-CKD patients than in their Western counterparts (14), the low frequency of these events may have been a factor contributing to this lack of a significant association. In fact, the incidence rate of CVD events (22.0 per 1,000 person-years) and all-cause death (19.8 per 1,000 person-years) in the present study were much lower than expected, possibly due to the small number of participants and the relatively short observation period. Further clinical studies with a larger sample size and longer followup period may reveal the association between serum inorganic phosphorus levels and these events in Japanese NDDCKD patients.

The association of higher serum inorganic phosphorus to adverse renal outcome may be explained by several mechanisms. First, in addition to direct phosphorus cytotoxicity, formation of calcium-phosphate crystals called calciproteins, a particle caused by an increased phosphorus load, can lead tubular injury, endothelial dysfunction, and vascular calcification in the kidneys (24). Second, increased phosphaturia can be related to renal damage. Decreased urinary reabsorption of phosphate was reportedly associated with a decreased eGFR and increased intact PTH value in NDD-CKD patients (25), and a recent study reported that elevated phosphaturia accelerated CKD progression due to renal tubular injury, even in the absence of hyperphosphatemia, in an animal model (26). Fibroblast growth factor 23 (FGF23), a peptide hormone inducing phosphaturia through its effects on proximal renal tubules, increases prior to the elevation of serum inorganic phosphorus levels in patients with NDD$\mathrm{CKD}$, maintaining the serum inorganic phosphorus levels in the reference range by promoting excretion via nephrons. Thus, increased phosphaturia may be involved in the mechanisms underlying the associations between serum inorganic phosphorus levels in the reference range and the occurrence of adverse kidney events.

An observational study reported that the administration of phosphorus binders was associated with reduced mortality in men with NDD-CKD (27). In a randomized control trial in NDD-CKD patients with serum inorganic phosphorus levels in the reference range, phosphorus binders significantly reduced serum inorganic phosphorus levels. In contrast, significant deterioration of vascular calcification was observed in the calcium-based phosphorus binder group (28). More 
recently, Toussaint et al. reported that treatment with lanthanum did not affect arterial stiffness or aortic calcification in patients with stage 3b/4 CKD who had normophosphatemia (29). Further studies will need to be conducted to determine the best strategy to prevent elevations in serum inorganic phosphorus levels during daily activities, such as dietary phosphorus restriction and the administration of phosphorus binders.

Several limitations associated with the present study warrant mention. First, only a small sample size was evaluated. The numbers of all-cause deaths and cardiovascular events were much lower than expected. Furthermore, due to limited number of patients, sub-analyses by sex were performed insufficiently in the present study. Thus, a larger sample size or a longer observation period will be needed to analyze the variables most strongly associated with all-cause death and cardiovascular events and investigate the differences by sex. Recently, $30 \%$ to $40 \%$ reductions in eGFR have been reported to be potentially useful surrogate endpoints in clinical research (30), and they might be suitable for determining renal disease progression in NDD-CKD patients, although these data were not available in the present study. Second, the serum inorganic phosphorus levels were only measured at baseline. Therefore, single measurements of serum inorganic phosphorus might have led to some misclassification of hyperphosphatemia or the serum inorganic phosphorus category. Further intervention studies addressing whether treatment to combat higher inorganic phosphorus level reduces the risk of CKD are necessary to clarify this issue. Third, we did not collect any data on the serum FGF23 levels, C-reactive protein (CRP) values, or medication use, such as phosphorus binders and activated vitamin $\mathrm{D}$, which can relate to both the serum inorganic phosphorus levels and outcomes in CKD patients. Fourth, the exclusion of many subjects without serum calcium and inorganic phosphorus data might have led to selection bias in the present study. These limitations of our present study will need to be addressed in future studies.

\section{Conclusions}

In summary, the present study showed that serum inorganic phosphorus levels were significantly associated with kidney events but not with all-cause death or cardiovascular events in Japanese NDD-CKD patients. Serum inorganic phosphorus levels of $\geq 3.7 \mathrm{mg} / \mathrm{dL}$ were related to a higher risk of CKD progression than levels of 2.9-3.2 mg/dL. Further studies will need to be conducted to determine why even serum inorganic phosphorus levels within the reference range can be associated with adverse outcomes in CKD and whether or not lowering the serum levels of inorganic phosphorus will delay CKD progression and improve the renal prognosis.

The authors state that they have no Conflict of Interest (COI).

\section{Acknowledgement}

The authors would like to thank Ayumi Kanno for her assistance in data collection.

\section{References}

1. Go AS, Chertow GM, Fan D, McCulloch CE, Hsu CY. Chronic kidney disease and the risks of death, cardiovascular events, and hospitalization. N Engl J Med 351: 1296-1305, 2004.

2. Block GA, Klassen PS, Lazarus JM, Ofsthun N, Lowrie EG, Chertow GM. Mineral metabolism, mortality, and morbidity in maintenance hemodialysis. J Am Soc Nephrol 15: 2208-2218, 2004.

3. Melamed ML, Eustace JA, Plantinga L, et al. Changes in serum calcium, phosphate, and PTH and the risk of death in incident dialysis patients: a longitudinal study. Kidney Int 70: 351-357, 2006.

4. Natoli JL, Boer R, Nathanson BH, et al. Is there an association between elevated or low serum levels of phosphorus, parathyroid hormone, and calcium and mortality in patients with end stage renal disease? A meta-analysis. BMC nephrol 14: 88, 2013.

5. Kestenbaum B, Sampson JN, Rudser KD, et al. Serum phosphate levels and mortality risk among people with chronic kidney disease. J Am Soc Nephrol 16: 520-528, 2005.

6. Schwarz S, Trivedi BK, Kalantar-Zadeh K, Kovesdy CP. Association of disorders in mineral metabolism with progression of chronic kidney disease. Clin J Am Soc Nephrol 1: 825-831, 2006.

7. Eddington H, Hoefield R, Sinha S, et al. Serum phosphate and mortality in patients with chronic kidney disease. Clin J Am Soc Nephrol 5: 2251-2257, 2010.

8. Kovesdy CP, Anderson JE, Kalantar-Zadeh K. Outcomes associated with serum phosphorus level in males with non-dialysis dependent chronic kidney disease. Clin nephrol 73: 268-275, 2010.

9. Chartsrisak K, Vipattawat K, Assanatham M, et al. Mineral metabolism and outcomes in chronic kidney disease stage 2-4 patients. BMC nephrol 14: 14, 2013.

10. Palmer SC, Hayen A, Macaskill P, et al. Serum levels of phosphorus, parathyroid hormone, and calcium and risks of death and cardiovascular disease in individuals with chronic kidney disease: a systematic review and meta-analysis. JAMA 305: 1119-1127, 2011.

11. Da J, Xie X, Wolf M, et al. Serum Phosphorus and Progression of CKD and Mortality: A Meta-analysis of Cohort Studies. Am J Kid Dis 66: 258-265, 2015.

12. Mehrotra R, Peralta CA, Chen SC, et al. No independent association of serum phosphorus with risk for death or progression to end-stage renal disease in a large screen for chronic kidney disease. Kidney Int 84: 989-997, 2013.

13. Menon V, Greene T, Pereira AA, et al. Relationship of phosphorus and calcium-phosphorus product with mortality in CKD. Am J Kidney Dis 46: 455-463, 2005.

14. Tanaka K, Watanabe T, Takeuchi A, et al. Cardiovascular events and death in Japanese patients with chronic kidney disease. Kidney Int 91: 227-234, 2017.

15. Inaguma D, Imai E, Takeuchi A, et al. Risk factors for CKD progression in Japanese patients: findings from the Chronic Kidney Disease Japan Cohort (CKD-JAC) study. Clin Exp Nephrol 21: 446-456, 2017.

16. Saito H, Tanaka K, Iwasaki $T$, et al. Xanthine oxidase inhibitors are associated with reduced risk of cardiovascular disease. Sci Rep 11: 1380, 2021.

17. Tanaka K, Saito H, Iwasaki T, et al. Association between serum potassium levels and adverse outcomes in chronic kidney disease: the Fukushima CKD cohort study. Clin Exp Nephrol 2021.

18. Janmaat CJ, van Diepen $M$, Gasparini A, et al. Lower serum calcium is independently associated with CKD progression. Sci Rep 8: 5148, 2018. 
19. Lim LM, Kuo HT, Kuo MC, et al. Low serum calcium is associated with poor renal outcomes in chronic kidney disease stages 34 patients. BMC nephrol 15: 183, 2014.

20. Voormolen N, Noordzij M, Grootendorst DC, et al. High plasma phosphate as a risk factor for decline in renal function and mortality in pre-dialysis patients. Nephrol Dial Transplant 22: 29092916, 2007.

21. Bellasi A, Mandreoli M, Baldrati L, et al. Chronic kidney disease progression and outcome according to serum phosphorus in mildto-moderate kidney dysfunction. Clinical journal of the American Society of Nephrology : Clin J Am Soc Nephrol 6: 883-891, 2011.

22. Chang WX, Xu N, Kumagai $\mathrm{T}$, et al. The Impact of Normal Range of Serum Phosphorus on the Incidence of End-Stage Renal Disease by A Propensity Score Analysis. PLoS One 11: e0154469, 2016.

23. McGovern AP, de Lusignan S, van Vlymen J, et al. Serum phosphate as a risk factor for cardiovascular events in people with and without chronic kidney disease: a large community based cohort study. PloS one 8: e74996, 2013.

24. Kuro OM. Phosphate as a Pathogen of Arteriosclerosis and Aging. J Atheroscler Thromb 2020.

25. Hong YA, Lim JH, Kim MY, et al. Assessment of tubular reabsorption of phosphate as a surrogate marker for phosphate regula- tion in chronic kidney disease. Clin Exp Nephrol 19: 208-215, 2015.

26. Santamaría R, Díaz-Tocados JM, Pendón-Ruiz de, Mier MV, et al. Increased Phosphaturia Accelerates The Decline in Renal Function: A Search for Mechanisms. Sci Rep 8: 13701, 2018.

27. Kovesdy CP, Kuchmak O, Lu JL, Kalantar-Zadeh K. Outcomes associated with phosphorus binders in men with non-dialysisdependent CKD. Am J Kidney Dis 56: 842-851, 2010.

28. Block GA, Wheeler DC, Persky MS, et al. Effects of phosphate binders in moderate CKD. Journal of the American Society of Nephrology : J Am Soc Nephrol 23: 1407-1415, 2012.

29. Toussaint ND, Pedagogos E, Lioufas NM, et al. A Randomized Trial on the Effect of Phosphate Reduction on Vascular End Points in CKD (IMPROVE-CKD). J Am Soc Nephrol 31: 2653-2666, 2020.

30. Matsushita K, Chen J, Sang Y, et al. Risk of end-stage renal disease in Japanese patients with chronic kidney disease increases proportionately to decline in estimated glomerular filtration rate. Kidney Int 90: 1109-1114, 2016.

The Internal Medicine is an Open Access journal distributed under the Creative Commons Attribution-NonCommercial-NoDerivatives 4.0 International License. To view the details of this license, please visit (https://creativecommons.org/licenses/ by-nc-nd/4.0/).

\section{(C) The Japanese Society of Internal Medicine} Intern Med Advance Publication 